\title{
Author Correction: Environmental stimuli drive a transition from cooperation to competition in synthetic phototrophic communities
}

Cristal Zuñiga (D), Chien-Ting Li(D), Geng Yu, Mahmoud M. Al-Bassam, Tingting Li, Liqun Jiang, Livia S. Zaramela, Michael Guarnieri, Michael J. Betenbaugh and Karsten Zengler (D)

Correction to: Nature Microbiology https://doi.org/10.1038/s41564-019-0567-6, published online 7 October 2019.

In the version of this Letter originally published, the first name of the author Tingting Li was spelt incorrectly as 'TingTing. This has now been corrected and the author initials updated accordingly in the Author contributions section.

Published online: 5 November 2019

https://doi.org/10.1038/s41564-019-0621-4

( The Author(s), under exclusive licence to Springer Nature Limited 2019 